\title{
CASCOM: Context-Aware Service Co-ordination in Mobile P2P Environments*
}

\author{
Heikki Helin ${ }^{1}$, Matthias Klusch ${ }^{2}$, António Lopes ${ }^{3}$, Alberto Fernández ${ }^{4}$, Michael \\ Schumacher ${ }^{5}$, Heiko Schuldt ${ }^{6}$, Federico Bergenti ${ }^{7}$, and Ari Kinnunen ${ }^{8}$ \\ 1 TeliaSonera Finland Oyj, Finland \\ Heikki.j.Helin@teliasonera.com \\ 2 Deutsches Forschungszentrum für Künstliche Intelligenz (DFKI), Germany \\ klusch@dfki.de \\ 3 Associação para o Desenvolvimento das Telecomunicações e Tecnicas de \\ Informática (ADETTI), Portugal \\ antonio.lopes@we-b-mind.org \\ 4 Universidad Rey Juan Carlos (URJC), Spain \\ alberto.fernandez@urjc.es \\ 5 Ecole Polytechnique Fédérale de Lausanne (EPFL), Switzerland \\ michael.schumacher@epfl.ch \\ 6 University for Health Sciences, Medical Informatics and Technology (UMIT), \\ Austria \\ heiko.schuldt@umit.at \\ 7 FRAMeTech S.R.L., Italy \\ bergenti@cs.unipr.it \\ 8 EMA Group, Ltd., Finland \\ ari@ema.fi
}

\begin{abstract}
The research project CASCOM (Context-aware Business Application Service Co-ordination in Mobile Computing Environments) will implement, validate, and trial value-added support for business services for mobile workers and users across mobile and fixed networks. The vision of the CASCOM approach is that ubiquitous application services are flexibly co-ordinated and pervasively provided to the mobile users by intelligent agents in dynamically changing contexts of open, large-scale, pervasive environments.
\end{abstract}

\section{CASCOM Overview}

The essential approach of the research project CASCOM is the innovative combination of agent technology, semantic Web services, P2P, and mobile computing for intelligent P2P (IP2P) mobile service environments. The services of our environment are provided by agents exploiting the coordination infrastructure to efficiently operate in highly dynamic environments. The IP2P infrastructure

\footnotetext{
* This work has been supported in part by the European Commission under the project grant FP6-IST-511632-CASCOM.
} 
includes efficient communication means, support for context-aware adaptation techniques, as well as dynamic service discovery and composition planning.

Software agents will be a key technology to address the challenges of our architecture. IP2P networks provide an environment for agents to collaborate as peers sharing information, tasks, and responsibilities with each other. Agents help to manage the P2P network complexity, and they will improve the functionality of conventional P2P systems. Our innovations in this domain will concern the development of context-aware agent-based semantic Web services, and flexible resource-efficient co-ordination of such services in the nomadic computing field. Further, context-awareness is investigated in the context of IP2P environment and we will develop context-aware agents which provide various business application services.

Service co-ordination mechanisms of P2P systems can be applied to multiagent systems to improve their efficiency. Although this may be accepted on a conceptual level, the combination of agents and P2P environments certainly deserves more innovative research, especially regarding nomadic environments. The dynamic topology of IP2P networks, characteristics of wireless network connections, and the limited capacity of mobile devices pose several challenges that have been addressed inadequately in service discovery architectures. In CASCOM, we will investigate mechanisms for service discovery algorithms for dynamic IP2P environments.

The problem of service co-ordination can be split into several sub problems: discovery, composition planning, execution monitoring, and failure recovery. CASCOM will advance the state of the art by carrying out innovative research on how these problems can be solved in IP2P environments. Especially CASCOM will provide flexible and efficient matching algorithms to be performed in large scale and resource limited IP2P environments.

Using AI planning formalisms in service composition and planning are developed for problems where the number of operators is relatively small but where plans can be complex. In Web service composition for open, large-scale IP2P environments planning methods dealing with huge number of possible service are required. However, plans are not necessarily very complex, and therefore planning methods must follow more closely the structure of the service directories. CASCOM will develop planning mechanisms that establish plan fragments directly on top of the service directory to solve this problem.

In general, it is expected that the outcomes of the project will have significant impact on the creation of a next-generation global, large-scale intelligent service environment. Both, research results on methods for service provision, discovery, composition and monitoring, and the deployed prototype of an open IP2P service environment in the context of nomadic computing will advance the state of the art of European and world knowledge in areas related to the deployment of services in open systems.

More information about CASCOM can be found from our homepage at http://www. ist-cascom.org/. 